\title{
Daueraufenthaltsstatus vs. öffentliche Sicherheit - Zum Rechtsrahmen der Ausweisung krimineller Drittstaatsangehöriger in der Europäischen Union
}

Kommentar zum Urteil des EuGH C-636/16 vom 7. Dezember 2017 (Lopez Pastuzano)

Sarah Progin-Theuerkauf *

Im Dezember 2017 entschied der EuGH im Fall Lopez Pastuzano, dass die Ausweisung von Drittstaatsangehörigen mit einer Daueraufenthaltsberechtigung (EU) nach der Richtlinie 2003/109/EG nur unter den Voraussetzungen von Art. 12 der Richtlinie möglich ist, d.h. die betreffende Person muss eine gegenwärtige, hinreichend schwere Gefahr für die öffentliche Ordnung oder die öffentliche Sicherheit darstellen und es muss stets eine individuelle Abwägung der Umstände des Einzelfalls durchgeführt werden. Ein Automatismus aufgrund einer strafrechtlichen Verurteilung ist unzulässig. Zudem spielt es nach der Richtlinie keine Rolle, ob die Ausweisung als verwaltungsrechtliche Sanktion ausgesprochen wurde oder Folge einer strafrechtlichen Verurteilung war.

I. Zum Sachverhalt 35

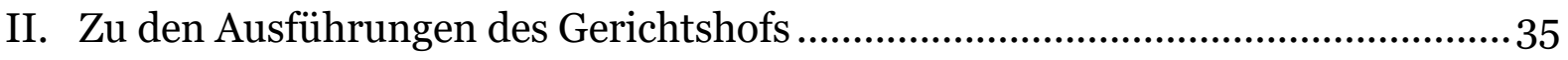

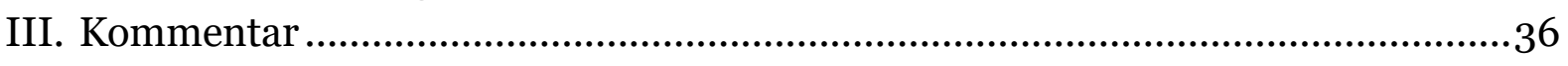

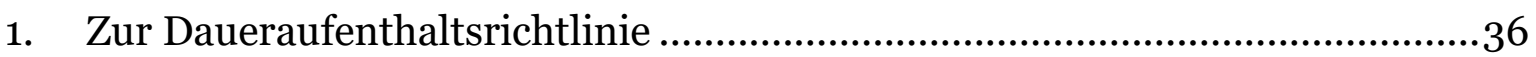

2. Zur Bedeutung des Urteils Lopez Pastuzano im Unionsrecht...........................38

3. Zu den Auswirkungen des Urteils auf die Schweiz .......................................39

Zitiervorschlag: Sarah Progin-Theuerkauf, Daueraufenthaltsstatus vs. öffentliche Sicherheit - Zum Rechtsrahmen der Ausweisung krimineller Drittstaatsangehöriger in der Europäischen Union, in: sui-generis 2018, S. 34

URL: $\quad$ sui-generis.ch/57

DOI: $\quad$ https://doi.org/10.21257/sg.57

* Prof. Dr. Sarah Progin-Theuerkauf ist Professorin für Europarecht und Migrationsrecht an der Universität Fribourg und Co-Direktorin des BeNeFri-Zentrums für Migrationsrecht. 


\section{Zum Sachverhalt}

1 Herr Lopez Pastuzano, ein kolumbianischer Staatsangehöriger, erhielt im Oktober 2013 in Spanien eine langfristige Aufenthaltserlaubnis (EU) nach der Richtlinie 2003/109/EG ${ }^{1}$ (Daueraufenthaltsrichtlinie). Im April 2014 wurde er zu zwei Freiheitsstrafen von zwölf bzw. drei Monaten verurteilt. Im Januar 2015 trat er seine Haftstrafe an, woraufhin ein Ausweisungsverfahren gegen ihn eingeleitet wurde. Im Juni 2015 erging eine Ausweisungsentscheidung, verbunden mit einem Einreiseverbot für 5 Jahre und dem Erlöschen der langfristigen Aufenthaltserlaubnis von Herrn Lopez Pastuzano. Hiergegen klagte dieser.

Nach spanischem Recht gibt es zwei Arten der verwaltungsrechtlichen Ausweisung: Eine Ausweisung, die als Strafe für bestimmte verwaltungsrechtliche Zuwiderhandlungen verfügt wird (Art. 57 Abs. 1 des Organgesetzes 4/2000), sowie eine Ausweisung als Rechtsfolge einer Verurteilung wegen einer vorsätzlichen Tat zu einer Freiheitsstrafe von mehr als einem Jahr (Art. 57 Abs. 2 des Organgesetzes 4/2000).

Art. 57 Abs. 5 des Organgesetzes 4/2000 setzt die Vorgaben von Art. 12 der Richtlinie 2003/109/EG in spanisches Recht um, wonach vor dem Erlass einer Verfügung über die Ausweisung die persönlichen Umstände der betroffenen Person zu berücksichtigen sind, d.h. die Dauer ihres Aufenthaltes in Spanien, Bindungen zu Spanien, Alter, Folgen für die betroffene Person und ihre Familienangehörigen

Richtlinie 2003/109/EG des Rates vom 25. November 2003 betreffend die Rechtsstellung der langfristig aufenthaltsberechtigten Drittstaatsangehörigen, ABl. L 16 vom 23. Januar 2004, S. 4453 . und Bindungen zum Staat, in den die Person zurückgeschickt werden soll. Dieser Artikel wurde von der Rechtsprechung in Spanien bislang überwiegend dahingehend ausgelegt, dass er nur für Ausweisungsverfügungen gelte, die als Strafe für verwaltungsrechtliche Zuwiderhandlungen erlassen wurden (also denjenigen nach Art. 57 Abs. 1), nicht aber bei Straftaten nach Art. 57 Abs. 2 des Organgesetzes. In letztgenannten Fällen findet daher nach herrschender Auffassung keine Abwägung der Umstände des Einzelfalls statt, die den Voraussetzungen von Art. 12 der Richtlinie genügen würde.

4 Da Herr Lopez Pastuzano zu einer Freiheitsstrafe von mehr als einem Jahr verurteilt worden war, ging die Behörde in der Entscheidung betreffend Herrn Lopez Pastuzano davon aus, dass Art. 57 Abs. 5 des Organgesetzes 4/2000 nicht anwendbar sei.

5 Das Verwaltungsgericht Nr. 1 Pamplona legte dem EuGH daher eine Frage nach der Auslegung von Art. 12 der Daueraufenthaltsrichtlinie und dessen Tragweite vor.

\section{Zu den Ausführungen des Gerichtshofs}

6 Der Gerichtshof stellte in seinem Urteil zunächst klar, dass es sich bei der vorgelegten Frage um eine zulässige Vorlagefrage handle (Rz. 16 bis 21). ${ }^{2}$ Die erbetene Auslegung stehe nämlich nicht «offensichtlich in keinem Zusammenhang mit der Realität oder dem Gegenstand des Ausgangsstreits» oder sei ein Problem «hypothetischer Natur». Die spanische Regierung hatte gerügt, es handle sich bei

2 Urteil des EuGH C-636/16 vom 7. Dezember 2017 (Lopez Pastuzano). 
der Vorlagefrage in Wahrheit um eine nach der Auslegung des spanischen Rechts, die nicht Gegenstand eines Vorabentscheidungsverfahrens nach Art. 267 AEUV vor dem EuGH sein könne. Diese Einschätzung teilte der Gerichtshof jedoch nicht. 3

7 In materieller Hinsicht äusserte sich der EuGH wie folgt:

8 Das vorrangige Ziel der Richtlinie 2003/109/EG sei die Integration von Drittstaatsangehörigen, die in den Mitgliedstaaten langfristig ansässig sind.4 Langfristig Aufenthaltsberechtigte sollten daher nach dem Willen des Unionsgesetzgebers einen verstärkten Ausweisungsschutz geniessen. Daher könnten Mitgliedstaaten nach Art. 12 der Richtlinie langfristig aufenthaltsberechtigte Drittstaatsangehörige nur dann ausweisen, wenn diese eine hinreichend schwere Gefahr für die öffentliche Ordnung oder die öffentliche Sicherheit darstellen.

9 Zudem bestimme Art. 12 Abs. 3 der Richtlinie, dass die Mitgliedstaaten vor der Verfügung einer Ausweisung gegen einen langfristig aufenthaltsberechtigten Drittstaatsangehörigen eine Abwägung vornehmen müssten; diese umfasse folgende Faktoren: die Dauer des Aufenthalts im Hoheitsgebiet, das Alter der betreffenden Person, die Folgen für die betreffende Person und ihre Familienangehörigen

3 Vgl. zum Gegenstand von Vorabentscheidungsverfahren statt vieler Astrid Epiney, in: Bieber/ Epiney/Haag/Kotzur, Die Europäische Union, 12. Auflage, 2016, § 9, Rz. 83, m.w.N. Die Frage der Zulässigkeit der Vorlage soll an dieser Stelle jedoch nicht weiter vertieft werden, da das materielle Recht im Zentrum dieses Beitrags steht.

4 Vgl. Urteile des EuGH C-508/10 vom 26. April 2012 (Kommission/Niederlande), Rn. 66 und C309/14 vom 2. September 2015 (CGIL und INCA), Rz. 21. sowie die Bindungen zum Aufenthaltsstaat oder fehlende Bindungen zum Herkunftsstaat. Es sei unerheblich, ob die Ausweisung als verwaltungsrechtliche Sanktion ausgesprochen werde oder Folge einer strafrechtlichen Verurteilung sei.

10 Der EuGH verwies sodann auf sein Urteil im Fall Ziebell5, in dem er 2011 bereits klargestellt hatte, dass eine Ausweisung nicht automatisch aufgrund einer strafrechtlichen Verurteilung verfügt werden könne, sondern dass stets eine Einzelfallprüfung vorzunehmen sei, die alle in Art. 12 Abs. 3 der Richtlinie genannten Gesichtspunkte abwäge.

11 Daraus folge, dass gegen einen langfristig aufenthaltsberechtigten Drittstaatsangehörigen nicht allein deshalb eine Ausweisungsentscheidung ergehen könne, weil er zu einer Freiheitsstrafe von mehr als einem Jahr verurteilt worden sei. Art. 12 stehe daher einer Regelung wie der spanischen entgegen, die - jedenfalls durch einen Teil der spanischen Gerichte - dahingehend ausgelegt und angewendet werde, dass sie nicht jede behördliche Ausweisungsverfügung gegen einen langfristig aufenthaltsberechtigten Drittstaatsangehörigen vom Vorliegen der in Art. 12 aufgestellten Voraussetzungen (insbesondere der Durchführung einer Abwägungsentscheidung unter Berücksichtigung der Umstände des Einzelfalls) abhängig mache.

\section{Kommentar}

\section{Zur Daueraufenthaltsrichtlinie}

12 Die Richtlinie 2003/109/EG (Daueraufenthaltsrichtlinie) ist Teil des unionsrechtlichen Acquis im Bereich der regulä-

5 Urteil des EuGH C-371/o8 vom 8. Dezember 2011 (Ziebell), Rz. 82 und 83. 
ren Einwanderung von Drittstaatsangehörigen (vgl. die Rechtsgrundlage in Art. 79 AEUV). Die Richtlinie aus dem Jahr 2003 war die erste, die den Rechtsstatus von Drittstaatsangehörigen unionsrechtlich regulierte, und ist damit auch die älteste in diesem Rechtsgebiet. Sie wurde 2011 dahingehend angepasst, dass auch Flüchtlinge und subsidiär Schutzberechtigte eine Daueraufenthaltsberechtigung (EU) erwerben können. ${ }^{6}$

Die Daueraufenthaltsrichtlinie verleiht langfristig Aufenthaltsberechtigten eine besonders geschützte, in der ganzen EU einheitliche Rechtsstellung. Dazu gehören u.a. Ansprüche auf Gleichbehandlung (Art. 11), eine beschränkte Freizügigkeit («kleine Freizügigkeit» bzw. «citizenship light»7) innerhalb der EU (Art. 14 und 15) und verbesserter Ausweisungsschutz (Art. 12). Die Aufenthaltserlaubnis für langfristig Aufenthaltsberechtigte nach der Richtlinie 2003/109/EG (langfristige Aufenthaltsgenehmigung-EG) ist unbefristet. Der Erwerb des Status eines langfristig Aufenthaltsberechtigten in der EU ist möglich, wenn sich ein Drittstaatsangehöriger fünf Jahre ununterbrochen rechtmässig auf dem Hoheitsgebiet eines Mitgliedstaates aufgehalten hat, über feste Einkünfte (ohne Inanspruchnahme von Sozialhilfeleistungen) verfügt und eine Krankenversicherung besitzt; er darf zu-

6 Richtlinie 2011/51/EU des Europäischen Parlaments und des Rates vom 11. Mai 2011 zur Änderung der Richtlinie 2003/109/EG des Rates zur Erweiterung ihres Anwendungsbereichs auf Personen, die internationalen Schutz geniessen, ABl. L 132 vom 19. Mai 2011, S. 1-4.

7 Vgl. Diego Acosta Arcazo, The Long-Term Residence Status as a Subsidiary Form of EU Citizenship - An Analysis of Directive 2003/109, Brill/Nijhoff 2011; Daniel Thym, EU Migration Policy and Its Constitutional Rationale: A Cosmopolitan Outlook, Common Market Law Review 50, no. 3 (2013), 709-736. dem keine Gefahr für die öffentliche Ordnung oder Sicherheit darstellen (Art. 4 bis 6 der Richtlinie).

14 Bei den anderen Instrumenten im Bereich der regulären Migration von Drittstaatsangehörigen handelt es sich um die Familienzusammenführungsrichtlinie 2003/86/EG8, die Blue-Card-Richtlinie 2009/50/EG9, die Richtlinie 2011/98/EU über eine kombinierte Erlaubnis ${ }^{10}$, die Saisonarbeiterrichtlinie 2014/36/EU11, die ICT-Richtlinie 2014/66/EU12 und die

8 Richtlinie 2003/86/EG des Rates vom 22. September 2003 betreffend das Recht auf Familienzusammenführung, ABl. L 251 vom 3. Oktober 2003, S. 12-18. Vgl. dazu Samah Posse-Ousmane/Sarah Progin-Theuerkauf, Le regroupement familial des ressortissants d'Etats tiers en Europe, Régimes juridiques et tendances actuelles, Jusletter 16. März 2015 .

9 Richtlinie 2009/50/EG des Rates vom 25. Mai 2009 über die Bedingungen für die Einreise und den Aufenthalt von Drittstaatsangehörigen zur Ausübung einer hochqualifizierten Beschäftigung, ABl. L 155 vom 18. Juni 2009, S. 17-29. Vgl. Samah Posse-Ousmane, Les conditions d'admission et de séjour des travailleurs hautement qualifiés dans l'UE, 2017.

10 Richtlinie 2011/98/EU des Europäischen Parlaments und des Rates vom 13. Dezember 2011 über ein einheitliches Verfahren zur Beantragung einer kombinierten Erlaubnis für Drittstaatsangehörige, sich im Hoheitsgebiet eines Mitgliedstaats aufzuhalten und zu arbeiten, sowie über ein gemeinsames Bündel von Rechten für Drittstaatsarbeitnehmer, die sich rechtmäßig in einem Mitgliedstaat aufhalten, ABl. L 343 vom 23. Dezember 2011, S. 1-9.

11 Richtlinie 2014/36/EU des Europäischen Parlaments und des Rates vom 26. Februar 2014 über die Bedingungen für die Einreise und den Aufenthalt von Drittstaatsangehörigen zwecks Beschäftigung als Saisonarbeitnehmer, ABl. L 94 vom 28. März 2014, S. 375-390. Dazu Margarite Helena Zoeteweij-Turhan, The Seasonal Workers Directive: '... but Some Are More Equal than Others', European Labor Law Journal 2017, Vol. 8, Issue 1, S. $28-44$.

12 Richtlinie 2014/66/EU des Europäischen Parlaments und des Rates vom 15. Mai 2014 über die Bedingungen für die Einreise und den Aufenthalt von Drittstaatsangehörigen im Rahmen eines unternehmensinternen Transfers, ABl. L 157 vom 27. Mai 2014, S. 1-22. Vgl. Sarah Progin-Theuerkauf, 
Richtlinie über Forscher und Studenten 2016/801/EU13.

Die genannten Richtlinien sind vor dem Hintergrund zu sehen, dass 2001 ein Versuch der EU-Kommission, die Arbeitsmigration von Drittstaatsangehörigen in die EU mithilfe einer transversalen Richtlinie $^{14} \mathrm{zu}$ regulieren, am Widerstand der Mitgliedstaaten scheiterte. Seither verläuft der Rechtsetzungsprozess in Bezug auf die Rechtsstellung Drittstaatsangehöriger eher schleppend. Die Kommission hat sich statt für ein transversales für ein sektorielles Vorgehen (ausgehend von bestimmten Kategorien von MigrantInnen) entschieden. ${ }^{15}$

Unternehmensinterne Transfers in der EU, Eine neue Form der Mobilität für Drittstaatsangehörige in der EU, Jusletter 14. März 2016.

13 Richtlinie (EU) 2016/801 des Europäischen Parlaments und des Rates vom 11. Mai 2016 über die Bedingungen für die Einreise und den Aufenthalt von Drittstaatsangehörigen zu Forschungs- oder Studienzwecken, zur Absolvierung eines Praktikums, zur Teilnahme an einem Freiwilligendienst, Schüleraustauschprogrammen oder Bildungsvorhaben und zur Ausübung einer Au-pair-Tätigkeit, ABl. L 132 vom 21. Mai 2016, S. 21-57. Die Richtlinie ersetzt zwei ältere Richtlinien von 2004 und 2005; vgl. Richtlinie 2004/114/EG des Rates vom 13. Dezember 2004 über die Bedingungen für die Zulassung von Drittstaatsangehörigen zur Absolvierung eines Studiums oder zur Teilnahme an einem Schüleraustausch, einer unbezahlten Ausbildungsmaßnahme oder einem Freiwilligendienst, ABl. L 375 vom 23. Dezember 2004, S. 12-18; Richtlinie 2005/71/EG des Rates vom 12. Oktober 2005 über ein besonderes Zulassungsverfahren für Drittstaatsangehörige zum Zwecke der wissenschaftlichen Forschung, ABl. L 289 vom 3. November 2005, S. 15-22. Dazu Samah PosseOusmane, L'admission des chercheurs, étudiants, élèves, volontaires et stagiaires dans l'UE - Vers une Europe du savoir?, Jusletter 17. März 2014; Agne Vaitkeviciute, Migration and mobility of third country researchers and students in the European Union and Switzerland, Jusletter 13. Februar 2017.

14 Europäische Kommission, Vorschlag für eine Richtlinie über die Bedingungen für die Einreise und den Aufenthalt von Drittstaatsangehörigen zur Ausübung einer unselbständigen oder selbständigen Tätigkeit, KOM (2001) 386 endg.

15 Vgl. dazu auch Samah Posse-Ousmane/Sarah Pro-
16 Dass eine verbesserte Rechtstellung von Drittstaatsangehörigen deren Integration fördert, wurde auf politischer Ebene erstmals 1999 im Rahmen des Europäischen Rates in Tampere ${ }^{16}$ festgehalten. In der Zwischenzeit ist das Ziel der «gerechteren Behandlung» von Drittstaatsangehörigen und die Annäherung ihrer Rechtsstellung an die der Unionsbürger allerdings etwas aus dem Fokus der Mitgliedstaaten verschwunden, da insbesondere die Asylmigration viel mehr Aufmerksamkeit erforderte. Insgesamt leben mehr als 7 Millionen daueraufenthaltsberechtigte Drittstaatsangehörige in der EU.17 Diese Zahl wird sich nach dem «Brexit» vermutlich noch erhöhen, da britische Staatsangehörige dann wohl als Drittstaatsangehörige gelten.

\section{Zur Bedeutung des Urteils Lopez Pastuzano im Unionsrecht}

17 Das Urteil Lopez Pastuzano ist bereits das elfte Urteil zur Auslegung der Daueraufenthaltsrichtlinie. ${ }^{18}$ Schon 2011 im Fall Ziebell19, den der EuGH auch im Urteil Lopez Pastuzano zitiert, war Art. 12 der Daueraufenthaltsrichtlinie Gegenstand eines Vorabentscheidungsverfahrens. Der

gin Theuerkauf, L'émergence d'une politique européenne de migration choisie, in: Institut für Europarecht (Hrsg.), Die Schweiz und die europäische Integration/La Suisse et l'intégration européenne, 20 Jahre Institut für Europarecht/20 ans de l'Institut de droit européen, 2015, S. 141-169; Thomas Gross/Alexandra Tryjanowski, Der Status von Drittstaatsangehörigen im Migrationsrecht der EU - Eine kritische Analyse, Der Staat, Vol. 48, No. 2, S. 259-277; Pieter Boeles/Maarten den Heijer/Gerrie Lodder/Kees Wouters, European Migration Law, 2nd edition, 2014, S. $127 \mathrm{ff}$.

16 Schlussfolgerungen des Vorsitzes, Europäischer Rat von Tampere, SN 200/99.

17 Vgl. Datenbank Eurostat zu asylum and managed migration.

18 Vgl. die regelmässig aktualisierte Liste des Zentrums für Migrationsrecht Nijmegen (NL).

19 Vgl. Fn. 5. 
EuGH betonte in Lopez Pastuzano einmal mehr, dass nach Art. 12 der Richtlinie stets eine individuelle Abwägung vorzunehmen ist, die der Situation des Betroffenen und der seiner Familienangehöriger Rechnung trägt. Eine einzige strafrechtliche Verurteilung ist nicht ausreichend. Auch Automatismen sind nicht zulässig. Die Rechtsnatur der zugrundeliegenden Ausweisungsentscheidung ist schliesslich unerheblich; diese kann verwaltungsrechtlichen oder strafrechtlichen Ursprungs sein.

Die Person muss zudem eine «hinreichend schwere» Gefahr für die öffentliche Ordnung oder die öffentliche Sicherheit darstellen; eine «einfache» Gefahr reicht somit nicht aus. Der EuGH macht hierzu allerdings keine weiteren Ausführungen, d.h. es bleibt offen, bei welchen Delikten eine «hinreichend schwere» Gefahr vorliegt.

19 Damit erfolgt eine Angleichung an die Rechtsstellung von Unionsbürgern, für die nach Art. 27 der Unionsbürgerrichtlinie $^{20}$ dieselben strengen Voraussetzungen gelten. Da es sich hier um Ausnahmen handelt, müssen diese laut EuGH restriktiv ausgelegt werden. ${ }^{21}$ Der Gerichtshof hat diese Grundsätze - vor allem im Urteil Donatella Calfa ${ }^{22}$ - bereits auf der Ba-

20 Richtlinie 2004/38/EG des Europäischen Parlaments und des Rates vom 29. April 2004 über das Recht der Unionsbürger und ihrer Familienangehörigen, sich im Hoheitsgebiet der Mitgliedstaaten frei zu bewegen und aufzuhalten, zur Änderung der Verordnung (EWG) Nr. 1612/68 und zur Aufhebung der Richtlinien 64/221/EWG, 68/360/ EWG, 72/194/EWG, 73/148/EWG, 75/34/EWG, 75/35/EWG, 90/364/EWG, 90/365/EWG und 93/96/EWG, ABl. L 158 vom 30. April 2004, S. $77-123$.

21 Urteil des EuGH C-145/09 vom 23. November 2010 (Tsakouridis); Urteil des EuGH C-249/11 vom 4. Oktober 2012 (Byankov).

22 Vgl. Urteil des EuGH C-348/96 vom 19. Januar sis der Grundfreiheiten aufgestellt, noch bevor diese in der Unionsbürgerrichtlinie kodifiziert wurden.

20 Auch in den anderen Rechtsakten des europäischen Migrationsrechts finden sich Bestimmungen zur öffentlichen Ordnung und Sicherheit. ${ }^{23}$ Es kann inzwischen von einer parallelen Auslegung dieser Begriffe in allen migrationsrechtlichen Instrumenten ausgegangen werden. ${ }^{24}$

\section{Zu den Auswirkungen des Urteils auf die Schweiz}

21 In der Schweiz gilt bei der Zulassung von AusländerInnen ein duales Zulassungssystem 25: UnionsbürgerInnen und ihre Familienangehörigen unterstehen dem Freizügigkeitsabkommen EU-CH26 (FZA). Drittstaatsangehörige sind hingegen nicht freizügigkeitsberechtigt; für sie gelten die restriktiven Regelungen des Ausländerge-

1999 (Donatella Calfa).

23 Vgl. zur Qualifikationsrichtlinie Urteil des EuGH C-373/13 vom 24. Juni 2015 (T.), zu diesem Thema zudem vertiefend Andrea Egbuna-Joss, Der Schutz der öffentlichen Ordnung und Sicherheit im Rahmen der internationalen Schutzgewährung: Eine Analyse der Qualifikationsrichtlinie 2011/95 der Europäischen Union unter besonderer Berücksichtigung der völkerrechtlichen Vorgaben. S. auch Sarah Progin-Theuerkauf, Unternehmensinterne Transfers in der EU, Eine neue Form der Mobilität für Drittstaatsangehörige in der EU, Jusletter 14. März 2016, Rz. 33 und Fn. 42. Zur Rückführungsrichtlinie vgl. zudem Urteil des EuGH C-554/13 vom 11. Juni 2015 (Zh. und O.).

24 Urteil des EuGH C-373/13 vom 24. Juni 2015 (T.), Rz. 77. Vgl. auch Constantin Hruschka/Sarah Progin-Theuerkauf, Die Rechtsprechung des EuGH zum Europäischen Migrationsrecht, in: Achermann et al. (Hrsg.), Jahrbuch für Migrationsrecht 2014/2015, Bern 2015, 273, 291.

25 Martina Caroni/Tobias Grasdorf-Meyer/Lisa Ott/ Nicole Schreiber, Migrationsrecht, 3. Auflage, 2014, S. $84 \mathrm{f}$.

26 Abkommen zwischen der Schweizerischen Eidgenossenschaft einerseits und der Europäischen Gemeinschaft und ihren Mitgliedstaaten andererseits über die Freizügigkeit vom 21. Juni 1999 (SR 0.142.112.681). 
setzes $^{27}$ (AuG), die in erster Linie die $\mathrm{Zu}-$ wanderung qualifizierter Arbeitskräfte ermöglichen (s. Art. 23 Abs. 1 AuG).

Da das Urteil Lopez Pastuzano die Daueraufenthaltsrichtlinie auslegt, die nicht Gegenstand eines bilateralen Abkommens ist, ist es für die Schweiz nicht verbindlich. Dennoch entfaltet es eine gewisse Signalwirkung: Denn auch im Freizügigkeitsabkommen findet sich - wie in Art. 27 der Unionsbürgerrichtlinie und in Art. 12 der Daueraufenthaltsrichtlinie eine Klausel, die eine Ausweisung von unter das Abkommen fallenden Personen aus Gründen der öffentlichen Ordnung, Sicherheit und Gesundheit erlaubt (Art. 5 Anhang I FZA ${ }^{28}$ ). Bei diesen Termini handelt es sich um unionsrechtliche Begriffe, für deren Auslegung nach Art. 16 Abs. 2 FZA die einschlägige Rechtsprechung des EuGH heranzuziehen ist. Insbesondere das EuGH-Urteil im Fall Donatella Calfa ${ }^{29}$ ist daher für die Schweiz verbindlich, da es vor Juni 1999 (Zeitpunkt der Unterzeichnung des Abkommens) erging. Auch nach dem FZA ist der behördliche Spielraum bei der Ausweisung von UnionsbürgerInnen daher begrenzt. So muss im Falle einer Ausweisung von straffällig gewordenen EU-BürgerInnen stets eine Einzelfallprüfung vorgenommen werden. ${ }^{30}$

27 Bundesgesetz über die Ausländerinnen und Ausländer vom 16. Dezember 2005 (SR 142.20).

28 Dieser lautet: «Die auf Grund dieses Abkommens eingeräumten Rechte dürfen nur durch Massnahmen, die aus Gründen der öffentlichen Ordnung, Sicherheit und Gesundheit gerechtfertigt sind, eingeschränkt werden.»

29 Vgl. Fn. 22. S. auch Urteil des Bundesgerichts 2C_108/2016 vom 7. September 2016, E. 2.3. m.w.N.

30 So auch Nina Burri/Valerio Priuli, Landesverweis und Freizügigkeitsabkommen, AJP 7/2017, S. 886 ff., 889 .
23 Das Urteil Lopez Pastuzano bestätigt schliesslich einmal mehr, dass die Umsetzung der Ausschaffungsinitiative ${ }^{31}$ in Art. 66a StGB, der seit dem 1. Oktober 2016 in Kraft ist, nicht unproblematisch ist, da sie eine obligatorische (d.h.) automatische Landesverweisung bei Vorliegen bestimmter Delikte (Katalogtaten) vorsieht. Die Vereinbarkeit der Landesverweisung mit dem FZA hängt nun entscheidend von der Anwendung der Härtefallklausel in Art. $66 a$ Abs. 2 StGB im Sinne der soeben besprochenen Rechtsprechung ab. ${ }^{2}$ Das Bundesgericht hat jedenfalls den unbedingten Vorrang des FZA vor (später erlassenem) innerstaatlichem Recht (und damit das Bestehen einer Ausnahme zur Schubert-Praxis) bereits bestätigt. 33

24 Konkrete Zahlen und Informationen über die Handhabung der Härtefallklausel sind bislang allerdings nicht verfügbar. 34

31 Vgl. Peter Uebersax, Zur Umsetzung der Ausschaffungsinitiative, ASYL 4/11, S. 9-13; Constantin Hruschka, Neue Gesetzesbestimmungen zur Umsetzung der Ausschaffungsinitiative, ASYL 4/16, S. 21-23. Vgl. auch Nina Burri/Valerio Priuli, Landesverweis und Freizügigkeitsabkommen, AJP 7/2017, S. 886; Marc Busslinger/Peter Uebersax, Härtefallklausel und migrationsrechtliche Auswirkungen der Landesverweisung, plädoyer 5/16, S. 100.

32 Vgl. zugunsten eines Vorrangs des FZA: Urteil des Obergerichts Zürich SB170250 vom 22. August 2017.

33 BGE 142 II 35 E. 3.2. f. und BGE 133 V 367, E 11.4 ff. $\mathrm{Zu}$ ersterem Urteil Astrid Epiney, Auslegung und Verhältnis des Freizügigkeitsabkommens zum nationalen Recht, Zum Urteil des Bundesgerichts 2C_716/2014 vom 26. November 2015, Jusletter 14. März 2016.

34 Vgl. Stefan Häne, Umstrittene Zahlen heizen Streit um kriminelle Ausländer an, Tagesanzeiger Online vom 16. Oktober 2017. 